\title{
The mass-metallicity relation of absorption selected high-redshift galaxies
}

\author{
Lise Christensen ${ }^{1}$, P. Møller ${ }^{2}$, Henrik Rhodin ${ }^{1}$, \\ Jens-Kristian Krogager ${ }^{1,3}$ and Johan P.U. Fynbo ${ }^{1}$ \\ ${ }^{1}$ Dark Cosmology Centre, Niels Bohr Institute, University of Copenhagen \\ Juliane Maries Vej 30, 2100 Copenhagen, Denmark, email: lise@dark-cosmology.dk \\ ${ }^{2}$ European Southern Observatory, Karl-Schwarzschildstrasse 2, D-85748 Garching bei \\ München, Germany \\ ${ }^{3}$ Institut d'Astrophysique de Paris, CNRS-UPMC, UMR 7095, 98bis Bd Arago, 75014, Paris, \\ France
}

\begin{abstract}
Strong absorption lines in quasar spectra primarily probe low-mass galaxies and detecting these in emission has previously been difficult. Dedicated surveys for the host galaxies of damped Lyman- $\alpha$ (DLA) systems have often resulted in non-detections and upper limits. Targeting the most metal-rich absorbers has proven to be a viable method, because these galaxies are brighter. By combining DLA metallicities and deriving host galaxy stellar masses, we find that metal-rich DLAs (with $>10 \%$ solar metallicity) and their host galaxies follow the same redshiftdependent scaling relation between stellar mass and metallicity as luminosity-selected galaxies. We derive a prediction for an absorber galaxy mass that depends on the DLA metallicity.
\end{abstract}

Keywords. galaxies: abundances, galaxies: high-redshift, quasars: absorption lines

\section{Introduction}

During the last decade large galaxy surveys have provided a wealth of information of the physical properties of thousands of galaxies at a range of redshifts. Observations have revealed relations between stellar masses and metallicities of galaxies (Tremonti et al. 2004), between stellar masses and star-formation rates (Noeske et al. 2007), as well as a fundamental relation that ties these three parameters together (Mannucci et al. 2010). Absorption-selected galaxies seem to trace a distinct, low-mass end of the galaxy population (e.g. Fynbo et al. 2008), where most galaxies lie below the detection limit of most surveys. Absorption-lines studies detect more metal-poor systems at intermediate redshifts, and derive metallicities at higher redshifts $(z>5$ in Rafelski et al. 2014; Hartoog et al. 2015) compared to galaxies observed in flux-limited surveys. Absorption line studies therefore provide a more comprehensive view of the metal-enrichment and chemical compositions in the high-redshift universe. In order to understand the connection between absorption- and emission-selected galaxies, we need to detect the absorbing galaxies in emission. Searches for the galaxy counterparts to damped Lyman- $\alpha$ absorbers (DLAs), have in several cases lead to upper limits only (e.g. Fumagalli et al. 2015). However, recent observations have demonstrated that metal-rich DLAs arise in more luminous galaxies (e.g. Fynbo et al. 2010; Krogager et al. 2012; Noterdaeme et al. 2012).

\section{Predicting absorbing galaxy masses}

We now finally know that the most metal-rich DLA galaxies are most likely to be detectable in emission. By connecting the velocity-metallicity relation for DLAs (Ledoux 
et al. 2006) with the redshift dependent mass-metallicity (MZ) relation for luminosityselected galaxies (Maiolini et al. 2008), Møller et al. (2013) derive a prediction of the galaxy stellar mass that depends only on the redshift and DLA metallicity.

We have verified this predicted relation directly in Christensen et al. (2014). Based on literature searches, we identified 12 confirmed galaxy - DLA pairs at $0.1<z<$ 3.2 with known galaxy redshifts corresponding to that of the DLA, impact parameters between $\sim 7$ and $40 \mathrm{kpc}$, and with measured DLA metallicities. From published multiband photometry (e.g. Rao et al. 2011), we derive the stellar mass of the spectroscopically confirmed DLA galaxy using standard spectral energy distribution (SED) fits. We then compare the stellar masses to the predicted masses. The offset between the predicted and measured stellar masses can be interpreted as an effect of a metallicity gradient, and the final absorption-line selected MZ relation can be expressed by the functional form:

$$
\log \left(M_{*} / \mathrm{M}_{\odot}\right)=1.76([\mathrm{M} / \mathrm{H}]+0.022 b+0.35 z+5.04),
$$

where $[\mathrm{M} / \mathrm{H}]$ is the DLA metallicity, $z$ is the redshift, and $b$ is the measured projected distance (in $\mathrm{kpc}$ ) between the QSO line of sight and the galaxy. The best fit metallic-

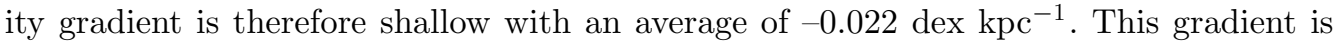
measured in the DLA galaxy outskirts, yet the value is in remarkable agreement with metallicity gradients measured directly within galaxy discs at $z \sim 1$ (Stott et al. 2014). The scatter around the MZ relation determined by Møller et al. (2013) is 0.67 dex in stellar mass, and by including a metallicity gradient the scatter reduces to 0.4 dex.

\section{Results: mass-metallicity relation of DLA galaxies}

After decades of trying to understand the nature of absorption selected galaxies, we are now at a stage where we can begin to explore scaling relations in absorption-selected galaxies. The main question to address is if DLA galaxies obey the same scaling relations as luminosity-selected galaxies. Fig. 1 shows the DLA metallicity as a function of the stellar mass for our current small sample, which includes the 12 pairs from Christensen et al. (2014) plus 8 more recent confirmed pairs (Straka et al. 2016, Rhodin et al. in prep). We have split up the samples in two redshift bins, one at $z<1$ (blue squares) and the other presenting $z>2$ DLA galaxies (red circles). In the left panel it is seen that most galaxies, both in the $z<1$ and $z>2$ samples, lie below their respective MZ relations for luminosity-selected galaxies (Savalio et al. 2005; Maiolino et al. 2008). Since impact parameters are known, we can use the information to correct for the effect that since DLA lines of sight trace the outskirts of galaxies, DLAs are more metal-poor compared to what would be measured for the galaxies themselves in emission. The right hand panel illustrates this correction where the red circles now follow the MZ relation, while the blue squares fit well at low stellar masses. At high stellar masses $\left(\log M_{*}>10\right)$, the metallicities appear over-corrected. This occurs because several galaxies are found at projected distances larger than $20 \mathrm{kpc}$, and the predicted metallicity offsets are higher than in reality. Metallicity gradients in local galaxies are steeper in their discs while it flattens to zero in the outskirts (Sánchez et al. 2014). This flattening at roughly two times the galaxy effective radius should be taken into account for the DLA galaxies metallicity correction, but this requires an examination of the DLA galaxies morphologies.

\section{Summary}

Given that DLA galaxies follow the redshift-dependent MZ relation just like other luminosity-selected galaxies, we now know that a metal-rich DLA must arise from a 

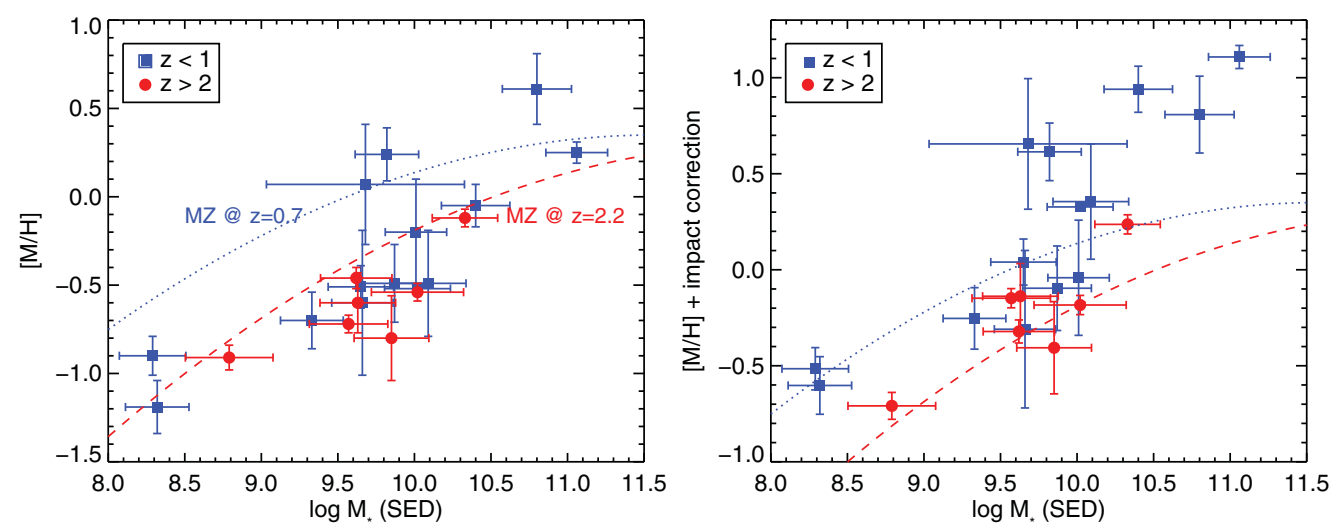

Figure 1. The right panel illustrates metallicities for DLAs at $z<1$ (blue squares) and $z>2$ (red circles) versus the stellar masses of the host galaxies. Known mass-metallicity relations for luminosity-selected galaxies at $z=2.2$ and $z=0.7$ are shown by the red and blue lines, respectively. Taking into account the DLA galaxy impact parameters and a gradient of -0.022 dex/kpc, we can correct the DLA metallicities to that predicted for the galaxies in emission (right panel). It is seen that the samples split up into their respective mass-metallicity relations.

relatively massive galaxy. Conventional arguments that when a galaxy is found at a large impact parameter of a few tens of $\mathrm{kpc}$, it cannot be the host galaxy of a DLA, and that the real absorbing galaxy is rather an undetected dwarf galaxy located right in the line of sight towards the QSO, can therefore be disregarded. The existence of an MZ relation for absorption selected galaxies also implies that the large spread of DLA metallicities measured at any redshifts to some extent reflects the MZ relation of the host galaxies, and that a range of galaxy stellar masses are probed by strong QSO absorption lines.

We stress that this study is based on metal-rich DLAs with metallicities $>10 \%$ solar. Extrapolating the study to the majority of the DLA population at $z \sim 2$, which has an average metallicity of $\sim 3 \%$ solar, implies an average galaxy stellar mass of $\sim 10^{8} \mathrm{M}_{\odot}$, which is below the detection limit of most existing deep imaging surveys. Verifying whether this extrapolation is valid will be possible with future extremely large telescopes.

\section{References}

Christensen, L., Møller, P., Fynbo, J. P. U., \& Zafar, T. 2014, MNRAS, 445, 225

Fumagalli, M., O’Meara, J. M., Prochaska, J. X., et al. 2015, MNRAS, 446, 3178

Fynbo, J. P. U., Prochaska, J. X., Sommer-Larsen, J., et al. 2008, ApJ, 683, 321

Fynbo, J. P. U., Laursen, P., Ledoux, C., et al. 2010, MNRAS, 408, 2128

Hartoog, O. E., Malesani, D., Fynbo, J. P. U., et al. 2015, A\&SA, 580, A139

Krogager, J.-K., Fynbo, J. P. U., Møller, P., et al. 2012, MNRAS, 424, L1

Ledoux, C., Petitjean, P., Fynbo, J. P. U., Møller, P., \& Srianand, R. 2006, A\& A, 457, 71

Maiolino, R., Nagao, T., Grazian, A., et al. 2008, A\&A, 488, 463

Mannucci, F., Cresci, G., Maiolino, R., Marconi, A., \& Gnerucci, A. 2010, MNRAS, 408, 2115

Møller, P., Fynbo, J. P. U., Ledoux, C., \& Nilsson, K. K. 2013, MNRAS, 430, 2680

Noeske, K. G., Weiner, B. J., Faber, S. M., et al. 2007, ApJL, 660, L43

Noterdaeme, P., Laursen, P., Petitjean, P., et al. 2012, A\& $A, 540$, A63

Rafelski, M., Neeleman, M., Fumagalli, M., et al. 2014, ApJL, 782, L29

Rao, S. M., Belfort-Mihalyi, M., Turnshek, D. A., et al. 2011, MNRAS, 416, 1215

Sánchez, S. F., Rosales-Ortega, F. F., Iglesias-Páramo, J., et al. 2014, A\&\&A, 563, A49

Stott, J. P., Sobral, D., Swinbank, A. M., et al. 2014, MNRAS, 443, 2695

Straka, L. A., Johnson, S., York, D. G., et al. 2016, MNRAS, 458, 3760

Tremonti, C. A., Heckman, T. M., Kauffmann, G., et al. 2004, ApJ, 613, 898 